\title{
Arterialization and anomalous vein wall remodeling in varicose veins is associated with upregulated FoxC2-DII4 pathway
}

\author{
Sumi Surendran ${ }^{1}$, Kalpana S Ramegowda², Aarcha Suresh', SS Binil Raj ${ }^{1}$, Ravi Kumar B Lakkappa ${ }^{3}$, \\ Giridhar Kamalapurkar², N Radhakrishnan ${ }^{4}$ and Chandrasekharan C Kartha ${ }^{1}$
}

Varicose veins of lower extremities are a heritable common disorder. Mechanisms underlying its pathogenesis are still vague. Structural failures such as valve weakness and wall dilatation in saphenous vein result in venous retrograde flow in lower extremities of body. Reflux of blood leads to distal high venous pressure resulting in distended veins. In an earlier study, we observed a positive association between c.-512C > T FoxC2 gene polymorphism and upregulated FoxC2 expression in varicose vein specimens. FoxC2 overexpression in vitro in venous endothelial cells resulted in the elevated mRNA expression of arterial endothelial markers such as Delta-like ligand 4 (DII4) and Hairy/enhancer-of-split related with YRPW motif protein 2 (Hey2). We hypothesized that an altered FoxC2-DII4 signaling underlies saphenous vein wall remodeling in patients with varicose veins. Saphenous veins specimens were collected from 22 patients with varicose veins and 20 control subjects who underwent coronary artery bypass grafting. Tissues were processed for paraffin embedding and sections were immunostained for DII4, Hey2, EphrinB2, $\alpha$-SMA, Vimentin, and CD31 antigens and examined under microscope. These observations were confirmed by quantitative real-time PCR and western blot analysis. An examination of varicose vein tissue specimens by immunohistochemistry indicated an elevated expression of Notch pathway components, such as DII4, Hey2, and EphrinB2, and smooth muscle markers, which was further confirmed by gene and protein expression analyses. We conclude that the molecular alterations in Dll4-Hey2 signaling are associated with smooth muscle cell hypertrophy and hyperplasia in varicose veins. Our observations substantiate a significant role for altered FoxC2-DII4 signaling in structural alterations of saphenous veins in patients with varicose veins.

Laboratory Investigation (2016) 96, 399-408; doi:10.1038/labinvest.2015.167; published online 25 January 2016

Distended and twisted veins in the legs known as varicose veins are a common venous disorder that can result in skin ulcers and venous thrombosis. They are characterized by reflux, incompetent venous and vein wall dilation. Progressive architectural remodeling of the venous vessel wall contributes to the pathophysiology of varicose veins and its complications. Epidemiological studies have demonstrated that varicose veins have a definite hereditary nature of inheritance. ${ }^{1,2}$ In conjunction with the presence of genes conferring disease susceptibility, various risk factors, such as prolonged standing, an increased body mass index, and preganancy, increases the mean venous pressure, in the lower extremities. ${ }^{3,4}$ Pfisterer et al in 2014 found in an experimental mouse model that an escalated venous filling pressure induces varicose-like venous remodeling. This process to a greater extent mimics detrimental remodeling processes observed in human varicose veins. ${ }^{5}$ The precise molecular mechanisms underlying the pathogenesis and progression of varicose veins are unclear. We have earlier observed a significant association of FoxC2 c. $-512 \mathrm{C}>\mathrm{T}$ polymorphism with the presence of varicose veins in patients. FoxC2 was also upregulated at both transcript and protein levels in varicose vein tissues of patients with varicose veins. ${ }^{6}$ In venous endothelial cells transfected with FoxC2-overexpressing mammalian vectors, the presence of putative arterial endothelial markers Delta-like ligand 4 (Dll4) and Hairy/enhancer-of-split related with YRPW motif protein 2 (Hey2) were found.

\footnotetext{
Cardiovascular Diseases and Diabetes Biology, Rajiv Gandhi Centre for Biotechnology, Thiruvananthapuram, Kerala, India; ${ }^{2}$ Departments of Pathology \& Cardiovascular Surgery, Sri Jayadeva Institute for Cardiovascular Sciences and Research, Bangalore, Bangalore, India; ${ }^{3}$ Department of Vascular Surgery, Kempegowda Institute of Medical Sciences, Bangalore, Bangalore, India and ${ }^{4}$ St Thomas Institute of Research on Venous Diseases, Changanassery, Kerala, India

Correspondence: Professor CC Kartha, MD, FRCP, Cardiovascular Diseases and Diabetes Biology, Rajiv Gandhi Centre for Biotechnology, Thycaud, Thiruvananthapuram, Kerala 695014, India.

E-mail: cckartha@rgcb.res.in

Received 18 September 2015; revised 20 November 2015; accepted 23 December 2015
} 
Table 1 Summary of source, dilutions, localization of markers used for western blot and immunohistochemistry

\begin{tabular}{|c|c|c|c|c|c|}
\hline Marker & Source & Manufacturer; Cat. No & Western blot dilution & IHC dilution & Localization \\
\hline $\mathrm{D} \| 4$ & Rabbit & Abcam,UK; ab7280 & $1: 1000$ & $1: 1200$ & Membrane \\
\hline EphrinB2 & Rabbit & SantaCruz, USA;sc-15397 & $1: 300$ & $1: 100$ & Transmembrane \\
\hline$\alpha-S M A$ & Mouse & Abcam,UK; ab5694 & $1: 8000$ & $1: 6000$ & Cytoplasm \\
\hline GAPDH & Mouse & Abcam, UK; ab9484 & $1: 1000$ & - & Cytoplasm \\
\hline Polyclonal secondary antibody to Rabbit lgG-H\&L (HRP) & Goat & Abcam, UK; ab97051 & $1: 10000$ & - & - \\
\hline Polyclonal secondary antibody to Mouse lgG-H\&L (HRP) & Rabbit & Abcam, UK; ab97046 & 1:5000 & - & - \\
\hline Normal rabbit lgG & Rabbit & Abcam, UK; ab27478 & - & $1: 100$ & - \\
\hline
\end{tabular}

Cat. No, catalog number; IHC, Immunohistochemistry.

In the study reported here, we explored whether a FoxC2-driven Dll4-Hey2 angiogenic signaling cascade is stimulated in varicose veins. It is known that Dll4 overexpression can induce smooth muscle cell (SMC) hypertrophy associated with vasculogenesis of tumor vessels. ${ }^{7}$ SMC proliferation induced by $\mathrm{Dll} 4$ and Hey2 may lead to arterialization of the saphenous vein wall in patients with varicose veins. We examined the expression of Dll4 and Hey2 at both transcriptional and translational levels in varicose vein specimens. We also assessed in varicose veins, the expression pattern of EphrinB2, an arterial-specific marker acting downstream to FoxC2 and Dll4 (ref. 8) in embryonic vessel development and pathological angiogenesis.

\section{MATERIALS AND METHODS}

\section{Study Subjects and Specimen Collection}

Varicose vein tissue samples were collected from 22 patients admitted for operative treatment of varicose veins at the Kempegowda Institute of Medical Sciences, Bangalore, India. Control saphenous vein tissues were also collected from residual vein tissues of 20 patients who underwent coronary artery bypass graft surgery at the Sri Jayadeva Institute for Cardiovascular Sciences and Research, Bangalore, India. Our study was approved by the human ethics committees of Rajiv Gandhi Centre for Biotechnology, Thiruvananthapuram, and collaborating hospitals. Samples were collected after obtaining informed written consent from study subjects.

Diagnosis of varicose veins was based on physical examination and Doppler ultrasound test. Patients in our study were from CEAP (classification based on Clinical, Etiology, Anatomy, Pathophysiology) Class 4, 5, and 6, and had varicose veins with various clinical signs such as pigmentation and ulceration along with pedal edema. Patients with venous obstructions from neoplasms were excluded from the study. We excluded patients with diabetes mellitus from our study as an earlier study reported that genetic variants of FoxC2 gene may give susceptibility to diabetes mellitus.

\section{Histopathologic Examination of Varicose Vein and Healthy Saphenous Vein Sections}

To analyze the venous structure by light microscopy, 22 varicose vein and 20 control saphenous vein samples were fixed in $4 \%$ formaldehyde and processed for paraffin embedding. Five-micrometer-thick sections were cut from the tissue blocks and sections were stained with hematoxylin and eosin (Sigma-Aldrich, USA).

\section{Immunostaining of DIl4, Hey2, EphrinB2, GAPDH,} $\alpha$-SMA, and CD31 Antigens in Human Tissue Specimens Paraffin-embedded tissue sections ( $5 \mu \mathrm{m}$ thick) were cut from 22 varicose vein tissue specimens and 20 control saphenous veins. Immunohistochemical staining for detecting Dll4, Hey2, EphrinB2, Vimentin, a-SMA, and CD31 in tissues was performed as described earlier. ${ }^{6}$ Details of primary and secondary antibodies used and their working dilutions are given in Table 1. After antibody incubation, DAB//Hydrogen Peroxide reaction system was used to visualize the immunostaining followed by nuclear staining using hematoxylin. Sections were washed in running water for adequate bluing, dehydrated, cleared, mounted in dibutyl-phthalate xylene, and observed under a light microscope (Nikon, Japan). Two sections of each sample were analyzed for the cellular architecture and staining intensity. An isotype control was performed to avoid any chances of non-specific staining.

\section{Image Processing and H Score Analysis}

Both hematoxylin and eosin and immunohistochemistry slides were observed under a Nikon Eclipse 55i microscopic system and acquired images were analyzed with Nikon NIS elements software (Japan). Five fields, which were 
Table 2 Primers used for real-time PCR in the study

\begin{tabular}{|c|c|c|c|}
\hline Gene & Primer sequence $5^{\prime} \rightarrow 3^{\prime}$ & AT $\left({ }^{\circ} \mathrm{C}\right)$ & $\begin{array}{l}\text { Amplicon } \\
\text { size (bp) }\end{array}$ \\
\hline \multirow[t]{2}{*}{ DI/4 } & F-ATGTGTCATTGCCACGGAGGTAT & 61 & 208 \\
\hline & R-AGGTGTGGAAGGGTATTGCATCTC & & \\
\hline \multirow[t]{2}{*}{ Hey2 } & F-GCAACAGGGGGTAAAGGCTAC & 63 & 335 \\
\hline & R-GAAGTTGTGGAGAGGCGAC & & \\
\hline \multirow[t]{2}{*}{ EphrinB2 } & F-TGGACAAGATGCAAGTTCTGCTGGA & 60 & 174 \\
\hline & R- CCGAGGATGTTGTTCCCCGA & & \\
\hline \multirow[t]{2}{*}{ CD31 } & F-GACAGTCAGAGTCATTCTTGCCCCA & 60 & 180 \\
\hline & R-CTCGTTGTTGGAGTTCAGAAGTGGT & & \\
\hline \multirow[t]{2}{*}{$\alpha-S M A$} & F-CACAACTGGCATCGTGCTGGAC & 60 & 169 \\
\hline & R-AGTAGTAACGAAGGAATAGCCACGC & & \\
\hline \multirow[t]{2}{*}{ Vimentin } & F-TGAACCTGAGGGAAACTAATCTGGA & 60 & 128 \\
\hline & R-ATCGTGATGCTGAGAAGTTCGTTG & & \\
\hline \multirow[t]{2}{*}{ GAPDH } & F-CCAGGCGCCCAATACGACCAA & 60 & 92 \\
\hline & R-TTCTTITGCGTCGCCAGCCGA & & \\
\hline
\end{tabular}

AT, Annealing temperature; bp, Base pairs; F, Forward; R, Reverse; $\alpha$, alpha.

randomly selected, were analyzed in all vein specimens. $\mathrm{H}$ score was calculated by multiplying the intensity of staining ( 0 for no staining, 1 for weak staining, 2 for medium and 3 for intense staining) with percentage of immunopositive cells. Calculated $\mathrm{H}$ score values were between 0 and 300 .

\section{Gene Expression Analysis of DIl4, Hey2, EphrinB2, Vimentin, $\alpha-S M A$, and CD31 in Varicose Vein Tissues by qRT-PCR}

Total RNA from all the 42 vein tissues $(100 \mathrm{mg})$ were extracted by All Prep DNA/RNA/Protein mini kit (Qiagen, USA). Quantification and purity of mRNA were measured by nanodrop-1000 spectrophotometer (ThermoScientific, USA) at $260 \mathrm{~nm}$. RNA was further treated with DNasel (Ambion, USA) for removing any DNA contamination.

Total RNA (500 ng) from each tissue sample was subjected to reverse transcription with oligodT, dNTPs, and M-MLV reverse transcriptase (Promega, USA). Primers for coding sequences of Dll4, Hey2, EphrinB2, GAPDH, a-SMA, and CD31 genes were designed for real-time PCR analysis (Table 2). Quantitative RT-PCR was carried out as reported earlier. ${ }^{6,9}$ Specific annealing temperatures for each primer sets and the expected amplicon size is given in Table 2 . Analysis was performed using ABI Prism 7900HT sequence detection system (Applied Biosystems, CA). Values were normalized with endogenous control GAPDH levels. Each analysis was performed in triplicates and with a minus-template negative control, which contained all the PCR components with water, as a template. A single peak in the dissociation curve for genes verified the specificity of amplified products.

\section{Protein Level Expression of DIl4, Hey2, EphrinB2, $\alpha$-SMA, Vimentin, and CD31 in Varicose Veins by Western Blot}

Proteins from tissue specimens were extracted with ice-cold RIPA buffer as described earlier. ${ }^{6}$ Proteins were estimated by using Bradford reagent (Bio-Rad, USA). Protein extracts $(25 \mu \mathrm{g})$ were subjected to $12 \%$ SDS-PAGE and western blot was performed. ${ }^{6}$ Details of primary and secondary antibodies used and their dilutions are given in Table 1. The membrane was re-probed with anti-GAPDH antibody (Abcam, UK) for normalization of protein expression. Densitometric analysis of immunoreactive bands was performed by Quantity One 1-D image analysis software program (Bio-Rad, USA).

\section{Statistical Analysis}

Normally distributed variables as in densitometric analysis of proteins are reported as mean \pm standard error, whereas non-normally distributed variables such as age are described by median values and interquartile range. We relied on the western blot densitometry rather than immunohistochemical scores from same samples for exact protein quantification mRNA expressions of each gene in individual control and test sample were calculated using formula $2^{-\Delta \Delta \mathrm{Ct}}\left(2^{\text {(Ct value of target gene-Ct value of control gene) }}\right)$. Fold change in mRNA expression in each sample was calculated against the mean expression in control vein samples for plotting individual samples' profiles. A column scatter plot was created using Graph Pad PRISM version 6.07. Student's $t$-test was used to analyze the difference in mRNA transcripts and protein expression levels. Differences between groups were considered significant for $P$ values less than 0.05 .

\section{RESULTS}

\section{Clinical Results}

The study population consisted of 22 patients with varicose veins (16 men and 6 women) and 20 control individuals (17 men and 3 women) who were patients with coronary artery disease and who underwent coronary artery bypass graft surgery. Median age of 22 patients with varicose veins was 36.5 years (range 21-65 years) and of the 20 control subjects was 49 years (range 40-72 years). None of the control subjects had clinical evidence of venous insufficiency or varicose veins.

Histopathology of Varicose and Control Vein Specimens Normal vein wall composed of three layers, intima, media, and adventitia. Tunica intima, which is nearest to the lumen, appeared thin, consisting of endothelium and subendothelial connective tissue. The media was formed of clusters of longitudinally oriented SMAs next to the intima. The adventitia consisted of SMCs, collagen fibers, and capillaries (Figure 1a). 

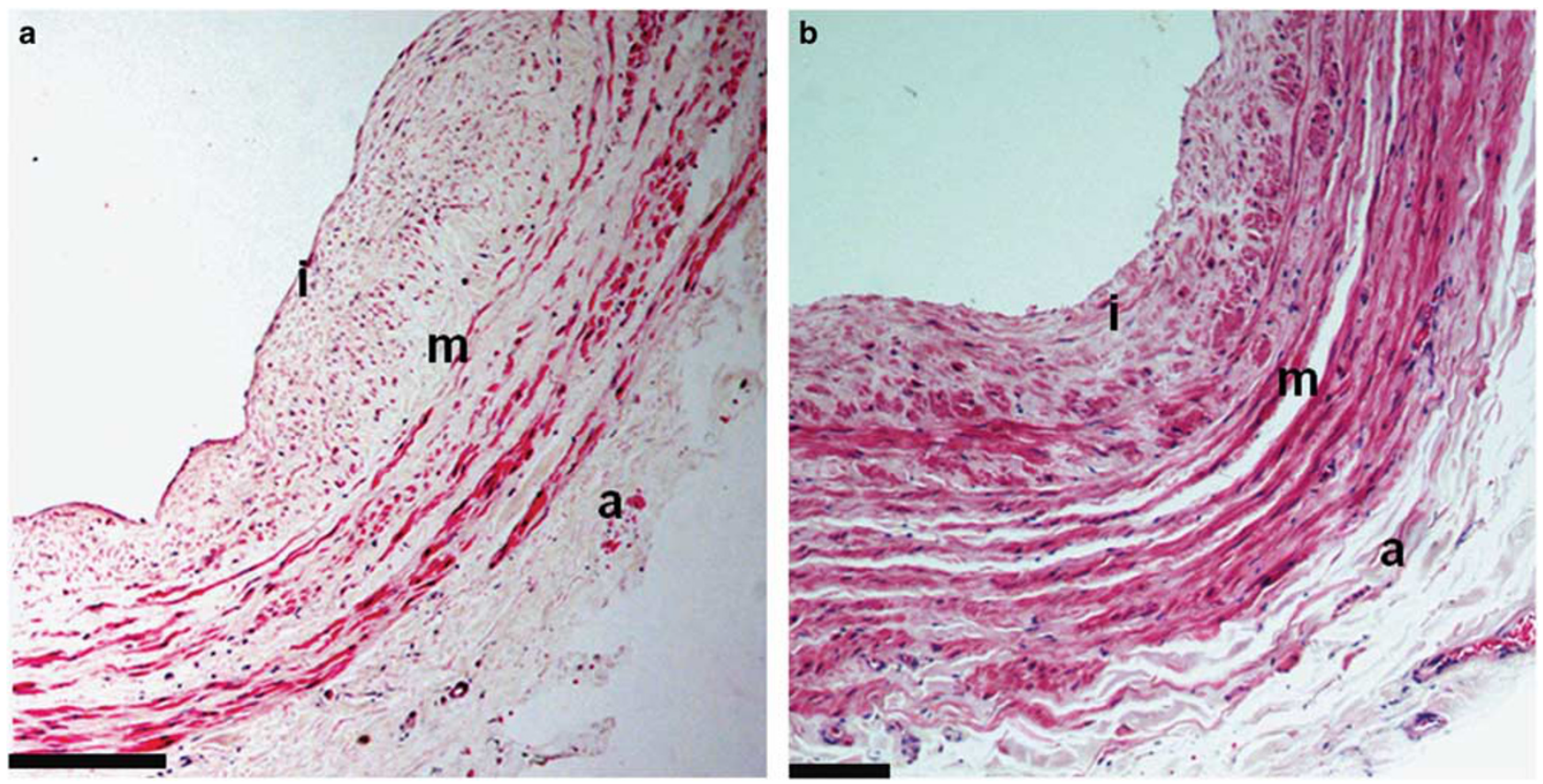

Figure 1 Photomicrograph (H\&E; x 10) of veins from a control subject (a) and a patient with varicose vein (b). Control saphenous vein has a well structured vein wall with normal thickness of all the three tunica (tunica intima (i), tunica media $(\mathrm{m})$ and tunica adventitia (a)). An abnormally thickened neointima (i), VSMC hypertrophy in media ( $\mathrm{m}$ ) and adventitia (a) are seen in the varicose vein sample. Scale bar equals $100 \mu \mathrm{m}$.

Varicose veins from all the patients had thickened vein wall. Endothelial lining of intima was discontinuous in most of the varicose vein samples. Thickness of the intima was significantly increased compared with normal veins (Figure 1b). In many cases, a 'neointima' formation was seen. Small bundles of SMCs were seen invading the media. Medial thickness was also significantly increased in varicose veins compared with normal veins. Clusters of circular and longitudinally oriented vascular SMCs (VSMC) were present in the media. Adventia was also seen to have larger number of SMCs in varicose veins.

\section{Vimentin and $\alpha$-SMA are Upregulated while CD31 is Downregulated in Varicose vs Control Tissues}

Vimentin and $a$-SMA were found to be increased in varicose veins specimens compared with control specimens (Figure 2), whereas CD31/PECAM-1 was found to be decreased in varicose veins. The difference in expression was statistically significant $(P<0.01)$ for all three genes.

Densitometry analysis of immunoblots also indicates Vimentin and $a$-SMA upregulation in varicose vein tissues compared with control tissues (Figure 3). CD31 was downregulated in varicose veins compared with control veins. CD31 protein levels were very less compared with mRNA levels in both varicose and non-varicose veins.

\section{Expression of $a$-Smooth Muscle Actin, Vimentin, and CD31 in Tissue Specimens}

Antibodies against $\alpha$-SMA and vimentin were used to demonstrate the presence of myofibrobalsts or SMCs.
$a$-SMA protein localized to the cell cytoplasm. Both anti- $\alpha$ SMA and anti-vimentin antibodies strongly stained the smooth muscle bundles in varicose veins and control veins. $a$-SMA was found expressed in the circular and longitudinal SMCs in the neointima and media of sections of varicose veins compared with only medial longitudinal SMC stacks in control vein sections (Figure 4a).

Vimentins are class-III intermediate filaments found in various non-epithelial cells, especially mesenchymal cells, such as myofibroblasts/SMCs. Vimentin staining was not as intense as $\alpha$-SMA staining in varicose veins in our study. Yet vimentin was found expressed in the SMCs of neointima, media, and adventia regions of tissue sections of varicose veins compared with medial longitudinal SMCs in control vein sections (Figure $4 \mathrm{~b}$ ).

CD31, also known as platelet endothelial cell adhesion molecule-1 (PECAM-1), is an integral membrane glycoprotein that is expressed at high levels in early endothelial cells. In 20 out of $22(91 \%)$ varicose vein specimens, CD31 immunostaining was virtually absent possibly due to denudation of the luminal endothelium during conventional stripping treatment. In all, 7 out of $20(35 \%)$ control specimens also had the loss of endothelial cells. CD31 staining was visible in endothelial cells of media and small capillaries in both test and control sections (Figure 4c). The overall expression of CD31 was low in varicose veins compared with controls. These differential expressions of proteins in varicose $v s$ control veins were further confirmed using $\mathrm{H}$ score analysis (Figure 5). 
a

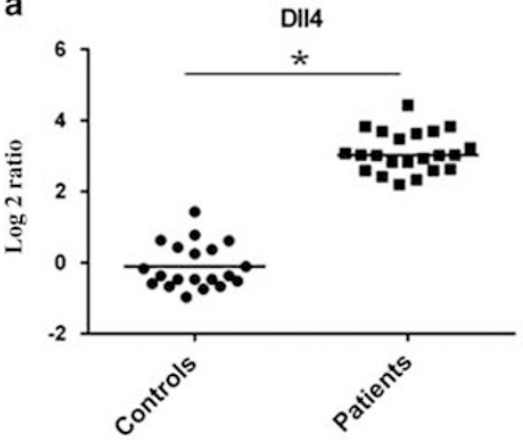

d

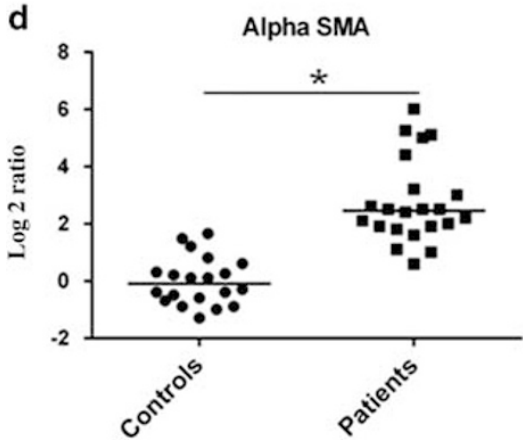

b

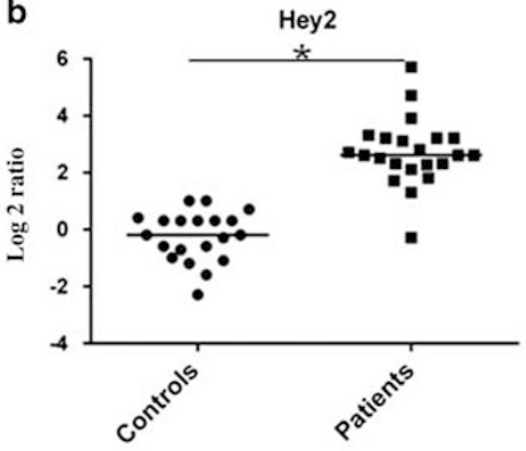

e

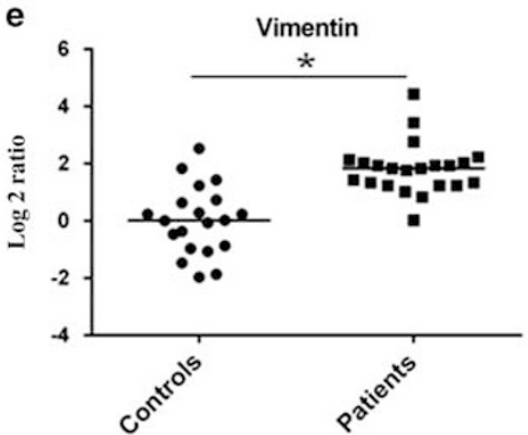

c

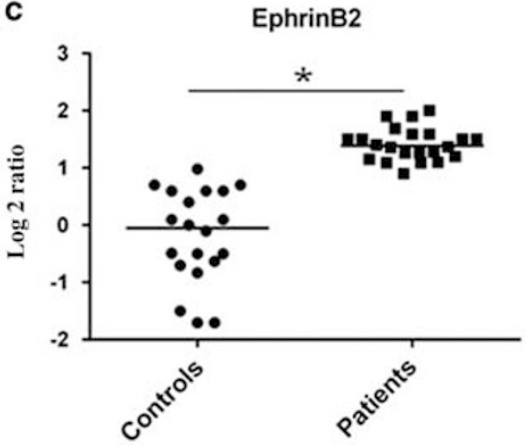

f

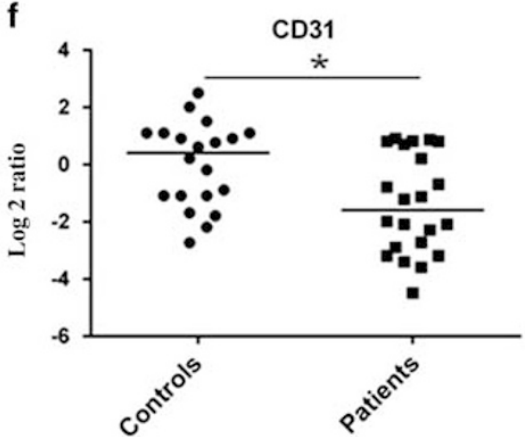

Figure 2 QRT-PCR analysis of putative genes in vein remodeling in varicose veins. Log2-transformed gene expression ratios obtained from QRT- PCR analysis in varicose $(n=22)$ and control veins $(n=20)$ are plotted for (a) DII4, (b) Hey2, (c) EphrinB2, (d) $\alpha$-SMA, (e) Vimentin, and (f) CD31. Each dot (control) and box (varicose) represents a data derived from one sample. For each sample, fold change in mRNA expression is calculated over its mean expression in control CABG vein samples. GAPDH was the endogenous calibrator. Median is denoted by the horizontal line.
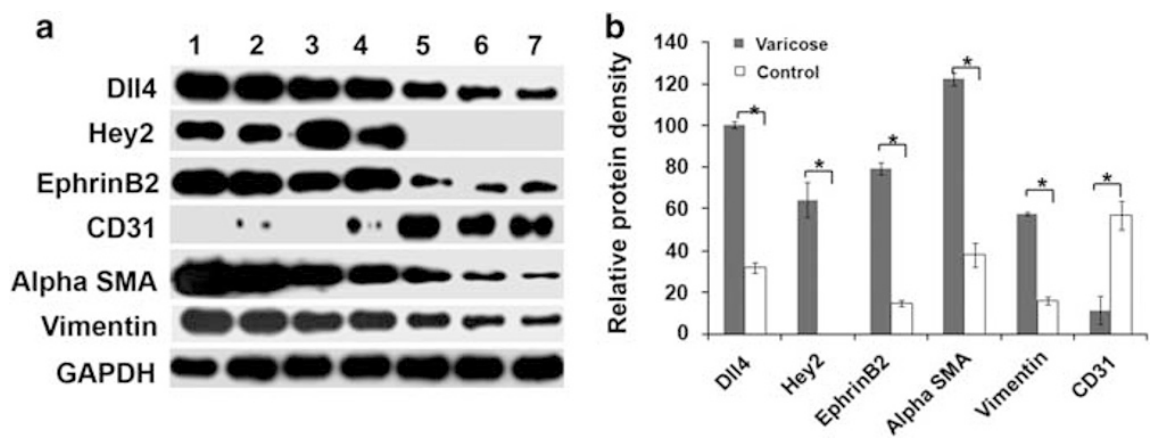

Figure 3 Western blot analysis of vein remodeling proteins in varicose vein tissues $(n=22)$ and control veins $(n=20)$. (a) Immunoblot analysis of DII4, Hey2, EphrinB2, $\alpha$-SMA, Vimentin, and CD31 proteins in patients and controls, lanes 1-4 patient vein tissue protein homogenates, lanes 5-7 normal saphenous vein protein homogenates. (b) Bar graph from densitometric analysis of immunoreactive bands of DII4, Hey2, EphrinB2, $\alpha$-SMA, Vimentin, and CD31 in varicose vein tissue specimens. DII4, Hey2, EphrinB2, $\alpha$-SMA, and Vimentin in varicose vein tissue specimens were highly upregulated compared with control veins. CD31 was low in varicose veins compared with control vein. Loading control was GAPDH. Standard error of mean in 22 patients are depicted as error bars. ${ }^{*} P<0.01$.

\section{Immunostaining of DII4, Hey2, and EphrinB2 Proteins in Vein Specimens}

The expression pattern for Hey2, Dll4, and EphrinB2 in the varicose veins and saphenous vein control samples were analyzed using immunostaining protocols. Dll4 was highly expressed in the neointima of all 22 varicose veins, whereas Dll4 staining was very mild in healthy vein specimens (Figure 6a). Expression of Dll4 was localized to intima and circular SMC bundles of neointima in varicose veins, compared with staining the in medial longitudinal SMC bundles in control veins.

Hey2 nuclear localization was observed in neointima and media of all the 22 varicose veins (Figure 6b), whereas Hey2 immunostaining was relatively absent in control saphenous veins. In three specimens, sparse staining was seen in medial SMCs. Hey2 was expressed in both endothelial cells and SMCs in the media of varicose veins. 


\section{CONTROL VEIN}

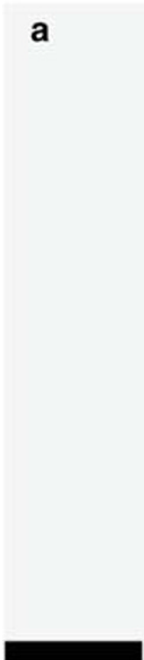

b
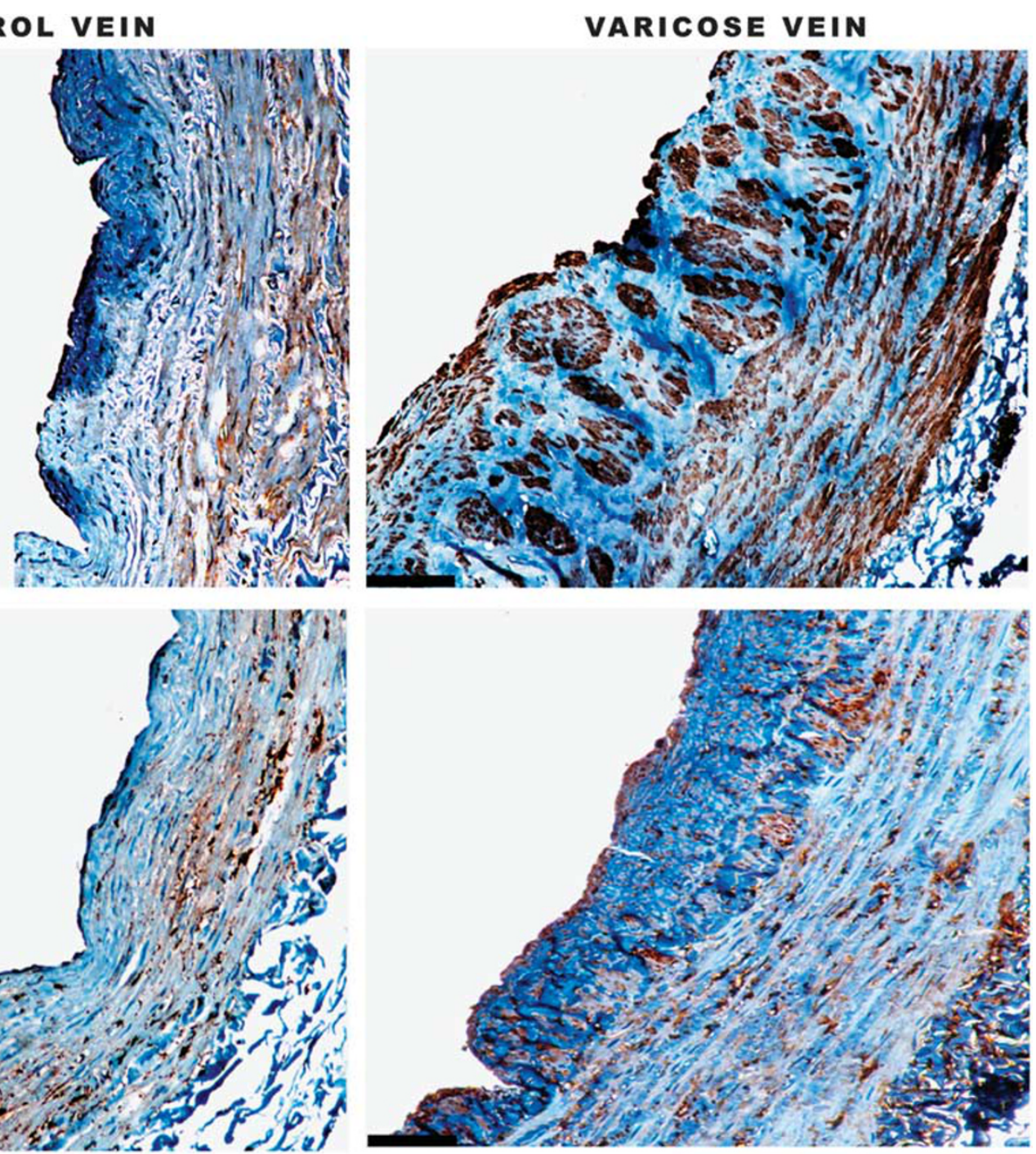

c
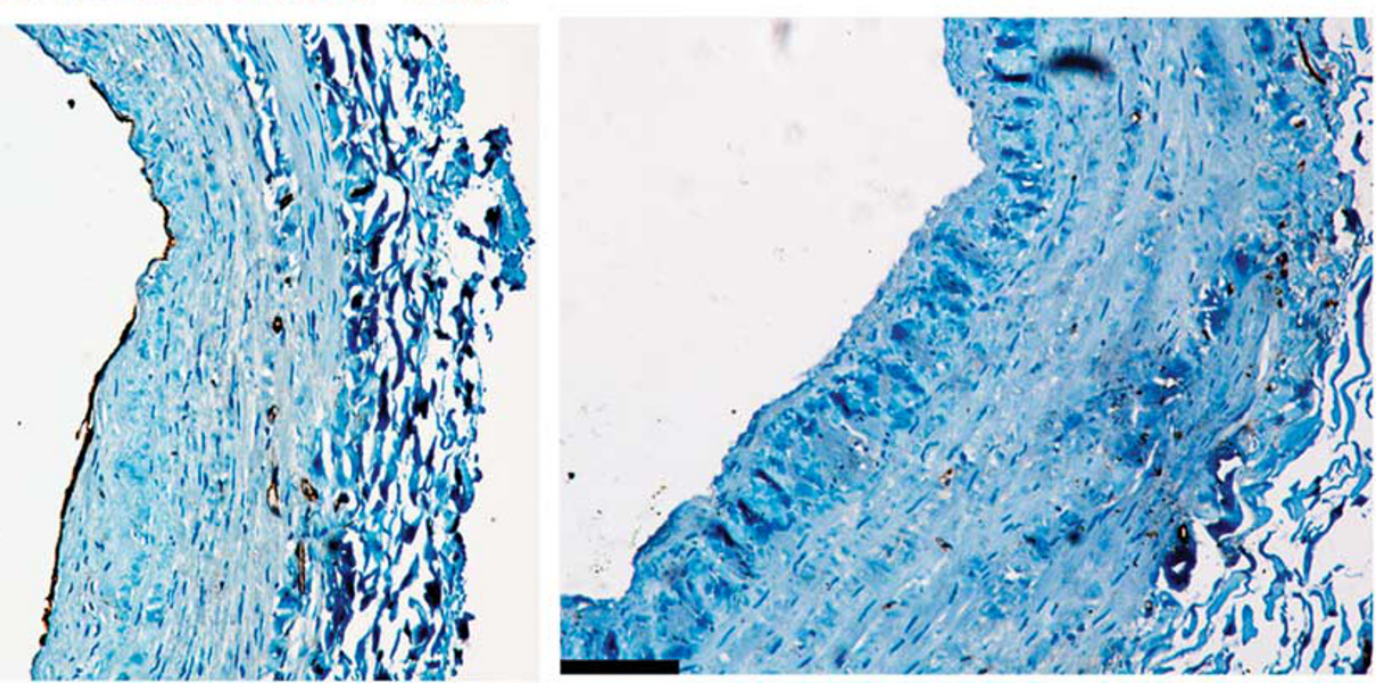

Figure 4 Photomicrograph of vein tissues stained with antibodies against (a) $\alpha$-SMA, (b) vimentin, (c) CD31 in control specimens and varicose veins (x10). There is intense staining for $\alpha$-SMA in the varicose vein specimens compared with control veins. Vimentin expression is localized to tunica media in control venous tissue section. Vimentin is overexpressed all over intima and media of varicose vein tissues with high staining intensity. CD31 expression is localized to endothelial lining in control venous tissue section. CD31 expression was not found in intimal lining in varicose veins as endothelial lining was found denuded. CD31 staining of endothelial cells is visible in media and toward adventitia, although in both control and varicose veins. Scale bar equals $100 \mu$ m. 


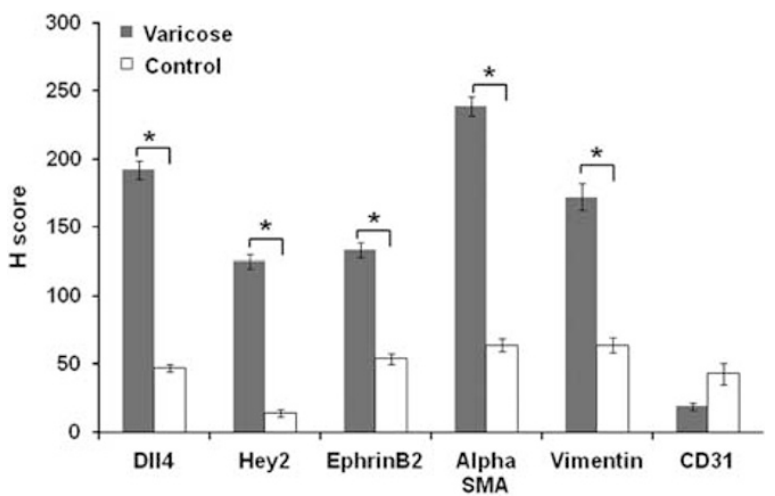

Figure 5 Semiquantitative analysis of vein remodeling proteins in immunostaining in varicose vein tissues $(n=22)$ and control veins $(n=20)$ using $\mathrm{H}$ score method. Dll4, Hey2, EphrinB2, $\alpha$-SMA, and Vimentin in varicose vein tissue specimens were highly upregulated compared with control veins. CD31 was less expressed in varicose veins compared with control vein. *Statistically significant $(P<0.05)$ when compared with control.

Immunostaining of EphrinB2 was meager in the endothelium and medial SMCs of normal saphenous veins. EphrinB2 was markedly overexpressed in both the endothelium and SMCs of neointima, media, and some regions of adventitia of varicose veins (Figure 6c). Expression patterns of proteins in varicose $v s$ control veins were quantitated manually using $\mathrm{H}$ score analysis (Figure 5).

Staining with rabbit and mouse IgG isotype control antibodies did not reveal positive staining in any of the sections, ruling out any chances of non-specific immunostaining. Representative images of negative immunostaining are given as Supplementary Figure 1.

\section{DII4, Hey2, EphrinB2 mRNA, and Protein Expression is Significantly Upregulated in Varicose Veins}

The expression pattern of Dll4, Hey2, and EphrinB2 was assessed in varicose vein tissues and control saphenous vein samples. Quantitative analysis was performed at both mRNA levels and protein levels by quantitative real-time PCR and western blot, respectively. The transcript levels of Dll4, Hey2, EphrinB2 genes were elevated in varicose vein samples when compared with control saphenous vein samples. In RNA samples from varicose vein tissue $(n=22)$, an overexpression of Dll4, Hey2, and EphrinB2 genes were found when compared with RNA extracted from non-varicose saphenous veins (Figure 2) and the increased expression was significant $(P<0.01)$.

Expression of Dll4, Hey2, and EphrinB2 proteins in varicose and non-varicose veins was assessed using western blot, with GAPDH as the loading control (Figure 3a). Densitometry analysis of immunoblots indicated a significant upregulation of Dll4, Hey2, and EphrinB2 proteins in varicose vein tissues when compared with control tissues (Figure 3b). Relative protein densities of Dll4, Hey2, EphrinB2, a-SMA and Vimentin in varicose vein tissue specimens were elevated compared with control veins and the difference was highly significant statistically $(P<0.01)$.

\section{DISCUSSION}

Varicose veins are known to be associated with progressive venous wall remodeling with SMC atrophy/hypertrophy and disturbed organization of extracellular matrix of veins. ${ }^{10}$ The precise molecular mechanisms involved in the vein wall remodeling in pathogenesis of varicose veins is unclear. Our study was prompted by our earlier observations in patients with varicose veins. A c. $-512 \mathrm{C}>\mathrm{T}$ polymorphism in the putative promoter region of FoxC2 gene is strongly associated with susceptibility to develop varicose veins. ${ }^{6}$ We also found that this variant increased the expression of FoxC2 in varicose vein tissues at both transcriptional and translational levels. FoxC2 upregulation in venous endothelial cells in vitro results in elevated mRNA expression of the arterial markers Dll4 and Hey 2 and downregulation of venous markers COUP-TFII and EphrinB4. We hence hypothesized that an altered FoxC2-Dll4 pathway is active in the pathologic remodeling in saphenous veins of patients with varicose veins.

\section{SMC Proliferation was Augmented in Varicose Veins Compared with Healthy Veins}

Histological analysis revealed an increase in intimal thickness in varicose veins compared with normal control veins. This increased thickness could be due to either an increase in SMCs or collagen content of intima. ${ }^{11}$ Other intimal changes include folding, invagination, fragmentation, and peeling of intimal endothelium. The infolding of the intimal layer of varicose vein wall results in a neointimal thickened region. Immunostaining with SMC markers such as $a$-SMA and vimentin $^{12}$ illustrated the infiltration of the neointima with SMCs. Immunostaining demonstrated marked alterations of $a$-SMA and vimentin staining confirming the increased mass of SMCs in varicose veins. In varicose vein tissues, medial SMCs were present in the form of longitudinally and circularly oriented bundles compared with both longitudinal bundles in normal saphenous veins. SMC infiltration was also seen in the adventitia of varicose veins.

Apart from being a putative myofibroblast marker, the higher expression of vimentin in varicose vein wall could also denote the transition of endothelial cells to SMC type. ${ }^{13}$ There are conflicting reports suggesting an increase ${ }^{14}$ and a decrease $\mathrm{e}^{11,15}$ of SMCs in varicose veins. In our subjects, we observed a marked increase in SMCs in the varicose vein wall.

Immunostaining with CD31 antibody to detect endothelial cells demonstrated a weak staining in varicose veins compared with healthy veins. The expression was too less compared with the mRNA levels of CD31 from the same tissues. A major extent of this lack of staining could be the result of conventional vein stripping surgery, which denudes the luminal endothelial layer. Another possibility is that being an early indicator of endothelial differentiation, ${ }^{16}$ 


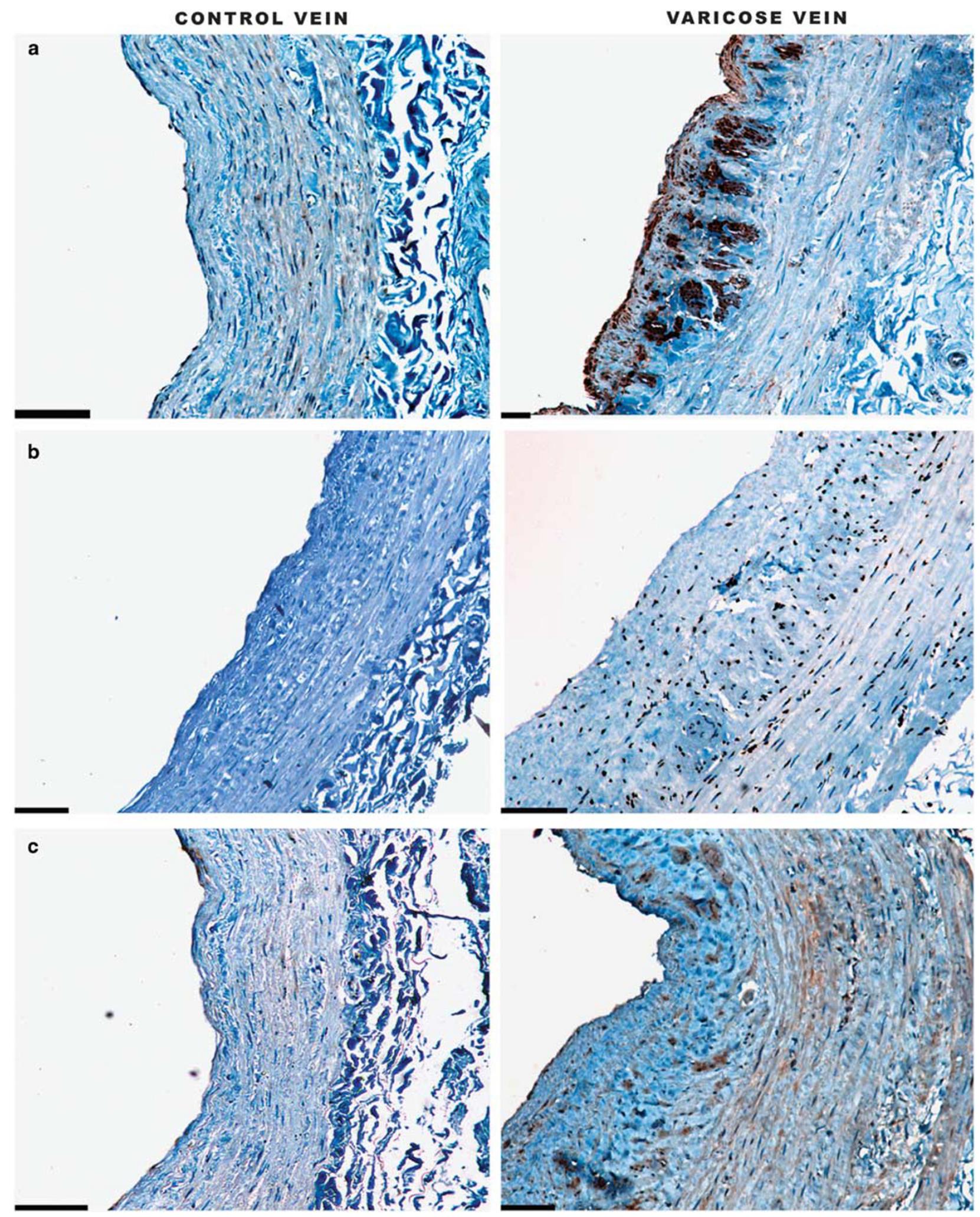

Figure 6 Photomicrograph of vein tissues stained with (a) DII4, (b) Hey2, and (c) ephrinB2 antibodies (x10). DII4 expression is localized to tunica media in control venous tissue section. Dll4 is expressed with high staining intensity all over the neointima of varicose vein tissues. Hey 2 was not expressed in control venous tissue section. Hey2 is seen expressed with high staining intensity all over the intima and media of varicose vein tissues. EphrinB2 expression is very weak in tunica media in normal veins. EphrinB2 is expressed all over the neointima and media of varicose veins. Scale bar equals $100 \mu \mathrm{m}$. 
CD31/PECAM-1 antigen disappears during the initial stages of endothelial mesenchymal transition in vascular pathological conditions.

\section{DII4- Hey2 Pathway is Upregulated in Varicose Veins}

We performed differential expression analysis for the downstream targets of FoxC2 such as Dll4 and Hey2 genes in 22 patients with varicose veins and 20 control specimens. After normalization with an endogenous calibrator GAPDH, there was a significant elevated expression of Dll4 and Hey2 in vein specimens of patients compared with control veins $(P<0.05)$. Significantly, in our earlier study, we had consistently observed FoxC2 overexpression in these same varicose vein specimens.

In an effort to localize these proteins in vein specimens, we performed immunohistochemical staining of sections with specific antibodies against Dll4 and Hey2 proteins. Dll4 was found overexpressed in the circular SMC bundles of neointima of varicosed sections, whereas its expression in control veins was weak. Hey2 was found expressed in the endothelial cells and SMCs of both neointima and media of varicose veins. We could not observe Hey2 staining in the control veins, except in three control veins where we found scattered and sparse staining in the media. Interestingly, we had earlier seen that FoxC2 is highly expressed in the neointima and media of varicose vein tissues compared with a mild expression only in the tunica media of control veins.

\section{FoxC2- DII4 -Hey2 Pathway is Associated with SMC Proliferation in Varicose Veins}

FoxC2 is a regulatory transcription factor involved in both lymphatic and vascular development. ${ }^{17}$ FoxC2 directly activate the Notch ligand Dll4 via a FoxC-binding site in the promoter of Dll4 gene. ${ }^{18}$ Earlier studies in vasculogenesis in the embryo ${ }^{19}$ and pathological angiogenesis in human tumors ${ }^{20}$ have delineated the role of Dll4 in arterial vessel development. Dll4 induces Notch signaling by activating Notch processing and liberate the intracellular domain. The released domain gets translocated to the cell nucleus and alters the expression of putative downstream target genes such as Hey 1 and 2. ${ }^{21}$ The Dll4-Hey2 pathway drives the uncontrolled VSMC accumulation during vascular remodeling, ${ }^{22}$ as observed in malformed vascular conditions such as varicose veins.

Upregulated Dll4 and Hey2 results in SMC proliferation. Although the overall quantity of SMCs in varicose veins remains high, the vascular wall in varicosities lacks a proper muscle contractile function. ${ }^{23}$ This may be because of the inability of SMCs to maintain necessary tone to the vein wall. ${ }^{24}$

\section{The Arterial Specific Marker EphrinB2 is Upregulated in Varicose Veins}

The observation that Dll4 is upregulated in varicose veins led us to examine other significant pathways in vascular wall development. The Dll4-Notch interaction is known to affect the interaction of ligand ephrinB2 with its receptor ephrinB4. ${ }^{25}$ EphrinB4 and EphrinB2 are specifically expressed in venous and arterial endothelial cells, respectively. ${ }^{26,27}$ In the adult human vasculature, ephrinB4 is expressed in both arteries and veins. EphrinB2 is, however, expressed solely in arterial endothelial cells even in adult human vessels. ${ }^{28}$ We had reported earlier that ephrinB4 is downregulated in varicose veins. ${ }^{6}$ Taking this cue, we assessed the expression pattern of ephrinB2 in our study specimens to look for any dysregulation in the ephrinB4/ephrinB2 axis in varicose veins. EphrinB2 was overexpressed in varicose veins compared with normal veins. Higher expression of ephrinB2 in varicose vein indicates a possible arterialization of the venous wall during varicose vein pathogenesis.

Limitations of the current study include the non-availability of tissues from patients with CEAP classes before $\mathrm{C} 4$, as compression therapy and minimal invasive treatments such as sclerotherapy and laser therapy are done in patients during these stages of the disease.

\section{CONCLUSION}

In summary, results of our study suggest a dysregulated FoxC2-Dll4 signaling in varicose veins. There is a decrease of venous markers such as COUP-TFII and EphrinB4 (ref. 6) and a markedly high expression of arterial markers such as FoxC2, Dll4, Hey2, and EphrinB2 in varicose veins implicating a non-physiological arterialization of saphenous veins in varicosities.

Earlier, $\mathrm{Tu}$ et al proposed that expression of arterial markers in arteriovenous malformations is due to the increased wall shear stress resulting from high-flow arteriovenous shunts. ${ }^{29}$ But another study by Diehl et al ${ }^{28}$ reported that in low-flow venous malformations also arterialization can occur, probably due to turbulent blood flow rather than arterial shear stress or tangential stress. Our findings indicate that similar mechanisms could contribute to the morphological alterations in varicose veins as well.

Supplementary Information accompanies the paper on the Laboratory Investigation website (http://www.laboratoryinvestigation.org)

\section{ACKNOWLEDGMENTS}

We thank Professor M Radhakrishna Pillai, Director, Rajiv Gandhi Centre for Biotechnology, Thiruvananthapuram, for providing funding and facilities for conducting this study.

\section{DISCLOSURE/CONFLICT OF INTEREST}

The authors declare no conflict of interest.

1. Cornu-Thenard A, Boivin P, Baud JM et al. Importance of the familial factor in varicose disease. Clinical study of 134 families. J Dermatol Surg Oncol 1994;20:318-326.

2. Fiebig A, Krusche $P$, Wolf $A$ et al. Heritability of chronic venous disease. Hum Genet 2010;127:669-674.

3. Sisto T, Reunanen A, Laurikka J et al. Prevalence and risk factors of varicose veins in lower extremities: mini-Finland health survey. Eur J Surg 1995;161:405-414. 
4. Bihari I, Tornoci L, Bihari P. Epidemiological study on varicose veins in Budapest. Phlebology 2012;27:77-81.

5. Pfisterer L, Meyer R, Feldner A et al. Bortezomib protects from varicoselike venous remodelling. FASEB J 2014;28:3518-327.

6. Surendran S, Girijamma A, Nair R et al. Forkhead box C2 promoter variant c. $-512 \mathrm{C}>\mathrm{T}$ is associated with increased susceptibility to chronic venous diseases. PLoS One 2014;9:e90682.

7. Schadler KL, Zweidler-McKay PA, Guan $\mathrm{H}$ et al. Delta-like ligand 4 plays a critical role in pericyte/vascular smooth muscle cell formation during vasculogenesis and tumor vessel expansion in Ewing's sarcoma. Clin Cancer Res 2010;16:848-856.

8. Hayashi $\mathrm{H}$, Kume T. Foxc transcription factors directly regulate DII4 and Hey2 expression by interacting with the VEGF-Notch signalling pathways in endothelial cells. PLoS One 2008;3:e2401.

9. Ramachandran S, Venugopal A, Sathisha K et al. Proteomic profiling of high glucose primed monocytes identifies cyclophilin $A$ as a potential secretory marker of inflammation in type 2 diabetes. Proteomics 2012;12:2808-2821.

10. Badier-Commander C, Couvelard A, Henin D et al. Smooth muscle cell modulation and cytokine overproduction in varicose veins. An in situ study. J Pathol 2001;193:398-407.

11. Travers JP, Brookes CE, Evans J et al. Assessment of wall structure and composition of varicose veins with reference to collagen, elastin and smooth muscle content. Eur J Vasc Endovasc Surg 1996;11:230-237.

12. Gabbiani G, Schmid E, Winter $S$ et al. Vascular smooth muscle cells differ from other smooth muscle cells: predominance of vimentin filaments and a specific alpha-type actin. Proc Natl Acad Sci USA 1981;78:298-302.

13. Mendez MG, Kojima S, Goldman RD. Vimentin induces changes in cell shape, motility, and adhesion during the epithelial to mesenchymal transition. FASEB J 2010;24:1838-1851.

14. Wali MA, Eid RA. Smooth muscle changes in varicose veins: an ultrastructural study. J Smooth Muscle Res 2001;37:123-135.

15. Krasinski $Z$, Kotwicka $M$, Oszkinis $G$ et al. Investigations on the pathogenesis of primary varicose veins. Wiad Lek 1997;50:275-280.

16. Kanayasu-Toyoda T, Yamaguchi T, Oshizawa T et al. CD31 (PECAM-1)bright cells derived from AC133-positive cells in human peripheral blood as endothelial-precursor cells. J Cell Physiol 2003;195:119-129.
17. Fauret $\mathrm{AL}$, Tuleja $\mathrm{E}$, Jeunemaitre $\mathrm{X}$ et al. A novel missense mutation and two microrearrangements in the FOXC2 gene of three families with lymphedema-distichiasis syndrome. Lymphology 2010;43:14-18.

18. Seo S, Fujita H, Nakano A et al. The forkhead transcription factors, Foxc1 and Foxc2, are required for arterial specification and lymphatic sprouting during vascular development. Dev Biol 2006;294:458-470.

19. Benedito $R$, Trindade A, Hirashima $M$ et al. Loss of Notch signalling induced by DIl4 causes arterial calibre reduction by increasing endothelial cell response to angiogenic stimuli. BMC Dev Biol 2008;8:117.

20. Ridgway J, Zhang G, Wu Y et al. Inhibition of Dll4 signalling inhibits tumour growth by deregulating angiogenesis. Nature 2006;444: 1083-1087.

21. Scehnet JS, Jiang W, Kumar SR et al. Inhibition of DII4-mediated signalling induces proliferation of immature vessels and results in poor tissue perfusion. Blood 2007;109:4753-4760.

22. Sakata $Y$, Xiang $F$, Chen $Z$ et al. Transcription factor $\mathrm{CHF} 1 / \mathrm{Hey} 2$ regulates neointimal formation in vivo and vascular smooth muscle proliferation and migration in vitro. Arterioscler Thromb Vasc Biol 2004;24:2069-2074.

23. Naim MM, Elsharaw MA. Histological assessment of the long saphenous vein in normal and varicose veins. Egyptian J Histology 2005;28:281-290.

24. Rose SS, Ahmed A. Some thoughts on the aetiology of varicose veins. J Cardiovasc Surg (Torino) 1986;27:534-543.

25. Duarte A, Hirashima $M$, Benedito $R$ et al. Dosage-sensitive requirement for mouse DII4 in artery development. Genes Dev 2004;18: 2474-2478.

26. Wang HU, Chen ZF, Anderson DJ. Molecular distinction and angiogenic interaction between embryonic arteries and veins revealed by ephrinB2 and its receptor Eph-B4. Cell 1998;93:741-753.

27. Gerety SS, Wang HU, Chen ZF et al. Symmetrical mutant phenotypes of the receptor EphB4 and its specific transmembrane ligand ephrin-B2 in cardiovascular development. Mol Cell 1999;4:403-414.

28. Diehl S, Bruno R, Wilkinson GA et al. Altered expression patterns of EphrinB2 and EphB2 in human umbilical vessels and congenital venous malformations. Pediatr Res 2005;57:537-544.

29. Tu J, Li Y, Hu Z. Notch1 and 4 signalling responds to an increasing vascular wall shear stress in a rat model of arteriovenous malformations. Biomed Res Int 2014;2014:368082. 